\title{
Clinical outcome of abdominal sacrocolpopexy in vault prolapse
}

\author{
Aparna Patil, Sheela HS, Madhva Prasad S, Samskruti Reddy
}

\begin{abstract}
Corresponding author: Dr. Aparna Patil, Assistant Professor, Department of Obstetrics and Gynecology, Vydehi Institute of Medical Sciences and Research Centre, Whitefield, Bangalore, India; Email : dr_aparna_patil@yahoo.com
\end{abstract}

Distributed under Attribution-Non Commercial - Share Alike 4.0 International (CC BY-NC-SA 4.0)

\begin{abstract}
Objectives: To describe the clinical outcome of patients undergoing abdominal sacrocolpopexy for posthysterectomy vault prolapse. Methods: This is a prospective observational study over a period of 2 years among patients who underwent the procedure. Demographic characteristics, POP-Q staging, intraoperative events, postoperative outcomes and complications were noted. Follow-up regarding outcome was done at 3 months, 6 months and 1 year. Results: 15 patients were studied with a mean age of 52 years and mean parity of 3 . Most of the patients had stage 3 or 4 vault prolapse. Intraoperative complications were encountered in 3 patients (one bladder injury, one bowel injury and one excessive hemorrhage) which were managed appropriately. On short term follow up one patient had dyspareunia and one patient had urinary dysfunction. Long term follow-up showed one patient having chronic pelvic pain. None of the patients had recurrence of vault prolapse. Conclusion: Abdominal sacrocolpopexy is a safe and an effective procedure for vault prolapse.
\end{abstract}

Keywords: Vault prolapse, posthysterectomy prolapse, abdominal sacrocolpopexy.

Vaginal vault prolapse is defined as descent of the vaginal cuff below a point that is $2 \mathrm{~cm}$ less than the total vaginal length above the plane of the hymen. ${ }^{1}$ The incidence of vault prolapse has increased as the average life expectancy has increased. The estimated life time risk of a woman undergoing surgery due to prolapse is $7 \% .^{2}$ The overall incidence of vault prolapse is estimated to be $0.2-1 \%$ after abdominal hysterectomy and $11.6 \%$ following vaginal hysterectomy. The most important risk factor for the development of vaginal vault prolapse is preoperative defect in the pelvic fascia which remains undetected and uncorrected at the time of hysterectomy. Apical prolapse can have a significant impact on the quality of life due to discomfort and associated bowel, bladder and sexual dysfunction. $^{3}$

The management of vaginal vault prolapse depends on the age of the patient, associated comorbidities, duration of anesthesia, desire to preserve sexual function and expertise of the surgeon. ${ }^{4}$ Conservative modalities like pessaries and pelvic floor exercises have limited role in management of vault prolapse. Various surgical methods, both vaginal and abdominal have been described over the years.

Vaginal procedures include sacrospinous ligament fixation, and uterosacral ligament fixation. Abdominal sacrocolpopexy is an abdominal procedure where the vaginal apex is suspended to the sacrum using a synthetic mesh. It restores the vaginal apex close to the normal anatomical position, approximately $1 \mathrm{~cm}$ anterior and $5 \mathrm{~cm}$ inferior to the

Received: $14^{\text {th }}$ July 2020. Peer review completed: $5^{\text {th }}$ August 2020, Accepted: $8^{\text {th }}$ August 2020.

Patil A, Sheela HS, Prasad S M, Reddy S. Clinical outcome of abdominal sacrocolpopexy in vault prolapse. The New Indian Journal of OBGYN. 2021; 7(2): 168-71. 
The New Indian Journal of OBGYN. 2021 (January-June);7(2)

$2^{\text {nd }}$ sacral vertebra. ${ }^{5}$

The aim of this study was to evaluate the anatomical and functional outcomes and complications during short term and long term follow up following abdominal sacrocolpopexy done for posthysterectomy vault prolapse at a tertiary care hospital.

\section{Materials and methods}

This was a prospective observational study conducted at Vydehi Institute of Medical Sciences and Research Center for a period of 2 years from 2017 to 2019. Institutional ethics committee approval was obtained. All patients who presented with posthysterectomy vault prolapse undergoing abdominal sacrocolpopexy were included in the study. After taking informed consent, thorough history taking and examination was performed. Baseline characteristics like age, parity, BMI, co-morbidities, previous surgery were noted.

Preoperative investigations like $\mathrm{CBC}$, blood grouping, FBS, PPBS, serology, RFT, LFT, urine routine microscopy, ECG and chest X-ray were done. Ultrasonography was done to rule out any pelvic pathology. Abdominal sacrocolpopexy was performed in all patients using synthetic mesh as per standard operative description below. Intra and postoperative complications were recorded.

Operative procedure details: Patient was placed in low lithotomy position. Low transverse laparotomy incision was made and from below a sponge stick was inserted in the vagina to manipulate the vault. Vaginal vault was held with long Allis forceps. Peritoneum was then dissected off the anterior vaginal wall. The peritoneum on the posterior vaginal wall was incised in the midline and carried down into the cul-de-sac. Synthetic mesh was cut in $\mathrm{Y}$ configuration. The double arm of $Y$ was used and 2-4 pairs of nonabsorbable sutures were placed anteriorly and 4-6 pairs of sutures were placed on the posterior vaginal wall. The peritoneum over the sacral promontory was opened and carried down over the anterior surface of the sacrum to expose the anterior longitudinal ligament where single arm of mesh was anchored. The peritoneum over the sacrum was then closed with 2-0, 3-0 absorbable sutures.

The vagina was inspected for any remaining defects. Posterior colporrhaphy and a perineoplasty were done in appropriate cases. The patients were followed up at 3 months, 6 months and 1 year after surgical procedure. During follow up data were collected regarding anatomical outcome or recurrence, complications, functional outcomes, urinary dysfunction, bowel disturbances and sexual dysfunction. Patients were subjected to repeat evaluation using the POP-Q system objectively. The collected data were noted, tabulated and presented below.

\section{Results}

During the study period of 2 years a total of 15 patients were included. Mean age of the study population was 52.2 years. Median parity was 3 . Out of 15 cases, 11 had a prior vaginal hysterectomy and 4 had prior abdominal hysterectomy. As per POP-Q classification, the distribution of patients is shown in table 1 .

\begin{tabular}{llll}
\multicolumn{4}{l}{ Table 1: Table showing stages as per POP-Q system } \\
\hline Stage & Vault prolapse & Cystocele & Rectocele \\
\hline Stage 1 & 0 & 3 & 4 \\
Stage 2 & 3 & 4 & 5 \\
Stage 3 & 6 & 8 & 6 \\
Stage 4 & 6 & - & - \\
Total & 15 & 15 & 15 \\
\hline
\end{tabular}

Major intraoperative complications encountered are shown in the table 2. One bladder injury occurred during dissection, during the attempt of separating the vault from the anterior vaginal wall. Injury was confirmed by retrograde filling of the bladder with methylene blue dye. The defect was repaired immediately and Foley's catheter was left in situ for 14 days. After removal, patient resumed smooth urinary function. One inadvertent serosal bowel injury occurred, which was identified intraoperatively and repaired with delayed absorbable sutures. Patient was kept nil per oral for 3 days, and there were no further problems. One patient had excessive haemorrhage during adhesiolysis which required only one unit blood transfusion postoperatively.

\begin{tabular}{ll} 
Table 2: Perioperative complications & \\
\hline Intra-operative complications & Number of patients \\
\hline Bladder injury & 1 \\
Bowel injury & 1 \\
Hemorrhage & 1 \\
\hline Postoperative complications & Number of patients \\
Pyrexia & 1 \\
Wound dehiscence & 1 \\
\hline
\end{tabular}

Postoperative complications included wound dehiscence in one patient due to poor diabetic control and postoperative pyrexia in one patient due to development of urinary tract infection (UTI).

Short term follow up at 3 months and 6 months showed dyspareunia in one patient and stress urinary incontinence in one patient. Long term follow up showed only one patient who had developed chronic pelvic pain. None of the patients had recurrence of vault prolapse, suggesting a $100 \%$ success rate of abdominal sacrocolpopexy at one year.

\section{Discussion}

Pelvic organ prolapse is a distressing condition which negatively affects the quality of life. Hence identification of vaginal defect and understanding pelvic anatomy is 
The New Indian Journal of OBGYN. 2021 (January-June);7(2)

important so that the management can be individualised. Choice of surgery depends on patient's age, co-morbid conditions, type of previous surgery and expertise of the surgeon. Many surgical approaches have been introduced to correct apical prolapse, however abdominal sacrocolpopexy has been widely studied and shown to be reliable. Abdominal sacrocolpopexy is an abdominal procedure in which a synthetic mesh is used to resuspend a prolapsed vaginal apex to the sacrum. The most commonly performed procedure is when the permanent mesh is attached to posterior vagina at the level of rectal reflection and to the anterior vagina for a distance of several centimetres.

Shika et al ${ }^{6}$ conducted a study in 2013 in Chandigarh, to evaluate the clinical outcomes after abdominal sacrocolpopexy (ASC). They found 100\% success rate of ASC at 1 year, which is similar to our study. One patient had hemorrhage and vault abscess. 1 had stress urinary incontinence (SUI) and 1 had severe pelvic pain due to sacral osteomyelitis. These findings are comparable to the results obtained in our study. Dhama et al ${ }^{7}$ have published a case series of abdominal sacrocolpopexy wherein the outcomes of 8 patients were reported, with no major complications. This study was very similar to ours.

A comprehensive review done by Nygaard et al ${ }^{8}$ about abdominal sacrocolpopexy showed it to be a reliable and effective procedure that could be used consistently to resolve vaginal vault prolapse. However, the minor risk associated for urinary and other complications needed to be taken into consideration. Success rate across different studies was 78$100 \%$. Mesh erosion rate across various studies was noted to be $3.4 \%$, but there was no such case reported in our study

Another retrospective study by Myung et al ${ }^{9}$ done from 1999-2003 in Seoul, Korea to assess the long-term outcome of abdominal sacrocolpopexy followed up over five years. In this study it was found a success rate of $86 \%$, and urinary dysfunction, bowel dysfunction and sexual dysfunction were significant adverse outcomes, and hence concluded that ASC has major complications risk which cannot be overlooked. These findings are in contrast to the results of our study which showed minimal urinary and bladder complications post-surgery in the follow up period.

Higgs et al ${ }^{10}$ did a prospective cohort study of 148 patients who have undergone ASC for vault prolapse. Success rate for surgery was $90 \%$ with $3 \%$ recurrence rate. $24 \%$ patients had SUI which required corrective surgery and $12 \%$ reported dyspareunia on long term follow up. A cochrane review ${ }^{11}$ included 3 randomized control trials
(RCT) compared abdominal sacrocolpopexy with sacrospinous fixation and meta-analysis showed that ASC was associated with lower recurrence rate, less postoperative SUI and decreased post op dyspareunia. A recent RCT by Nygaard et al has also showed that ASC is a good procedure for pelvic organ prolapse and there is reduction noted in stress urinary incontinence rates also, over a 7 year follow up period. ${ }^{12}$

To sum up, a recent systematic review meta-analysis also stated that no differences could be delineated between the various techniques available for the surgical management of vault prolapse. ${ }^{13}$ Many novel techniques such as laparoscopic and robotic sacrocolpopexy have evolved. Though laparoscopic sacrocolpopexy can be equally effective as ASC in selected women, inherent problems such as technical expertise and cost are disadvantages.

\section{Conclusion}

This study shows that on one year follow up, there is no persistence or recurrence of complaints at 1 year of procedure. Abdominal sacrocolpopexy appears to be a safe and effective procedure for treatment of posthysterectomy vaginal vault prolapse with minimal complications.

\section{Conflict of interest: None. Disclaimer: Nil.}

\section{References}

1. Haylen BT, Maher CF, Barber MD, Camargo S, Dandolu V, Digesu A, et al. An International Urogynecological Association (IUGA)/International Continence Society (ICS) joint report on the terminology for female pelvic organ prolapse (POP). International urogynecology journal. 2016 Feb 1; 27(2): 165-94.

2. Handa VL. Vaginal Vault Prolapse. In: Jones H, Rock J, editors. Te Linde's Operative Gynecology. 11th Edition. Philadelphia: Wolter Kluwer. 2015. pp 885-904.

3. Barrington JW, Edwards G. Posthysterectomy vault prolapse. International Urogynecology Journal. 2000 Aug 1; 11(4): 241-5.

4. Marchionni M, Bracco GL, Checcucci V, Carabaneanu A, Coccia EM, Mecacci F, et al. True incidence of vaginal vault prolapse. Thirteen years of experience. The Journal of reproductive medicine. 1999 Aug; 44(8): 679-84.

5. Benson JT, Lucente V, McClellan E. Vaginal versus abdominal reconstructive surgery for the treatment of pelvic support defects: a prospective randomized study 
The New Indian Journal of OBGYN. 2021 (January-June);7(2)

with long-term outcome evaluation. American journal of obstetrics and gynecology. 1996 Dec 1; 175(6): 1418-22.

6. Rani S, Pandher DK, Huria A, Mehra R. Clinical outcome of abdominal sacrocolpopexy. Journal of midlife health. 2015 Oct; 6(4):169-72.

7. Dhama V, Singh S, Chaudhary R, Bajwa H. Case series of abdominal sacral colpopexy. Int J Reprod Contracept ObstetGynecol. 2016; 5:1992-5.

8. Nygaard IE, McCreery R, Brubaker L, Connolly A, Cundiff G, Weber AM, et al. Abdominal Sacrocolpopexy: A comprehensive Review. Obstetrics and Gynecology. 2004. 104(4): 805-19.

9. Jeon MJ, Moon YJ, Jung JJ, Lim KJ, Yang HI, Kim SK, et al. A long-term treatment outcome of abdominal sacrocolpopexy. Yonsei Med J. 2009; 50(6): 807-13.

10. Higgs $\mathrm{P}$, Goh J, Krause $\mathrm{H}$, Sloane $\mathrm{K}$, Carey $\mathrm{M}$. Abdominal sacral colpopexy: an independent prospective long-term follow-up study. Aust N Z J Obstet Gynaecol. 2005 Oct; 45(5):430-4.

11. Maher C, Feiner B, Baessler K, Christmann-Schmid C, Haya N, Brown J. Surgery for women with apical vaginal prolapse. Cochrane Database Syst Rev. 2016;10(10): CD012376.
12. Nygaard I, Brubaker L, Zyczynski HM, Cundiff G, Richter H, Gantz M, et al. Long-term outcomes following abdominal sacrocolpopexy for pelvic organ prolapse. JAMA. 2013; 309: 2016-24

13. Coolen AWM, Bui BN, Dietz V, Wang R, van Montfoort APA, Mol BWJ, et al. The treatment of posthysterectomy vaginal vault prolapse: a systematic review and meta-analysis. Int Urogynecol J. 2017; 28(12):1767-83.

\footnotetext{
Aparna Patil ${ }^{1}$, Sheela $\mathrm{HS}^{2}$, Madhva Prasad $\mathrm{S}^{3}$, Samskruti Reddy ${ }^{4}$

${ }^{1}$ Assistant Professor, Department of Obstetrics and Gynecology, Vydehi Institute of Medical Sciences and Research Centre, Whitefield, Bangalore, India;

${ }^{2}$ Professor and Unit Head, Department of Obstetrics and Gynecology, Vydehi Institute of Medical Sciences and Research Centre, Whitefield, Bangalore, India; ${ }^{3}$ Associate Professor, Department of Obstetrics and Gynecology, Vydehi Institute of Medical Sciences and Research Centre, Whitefield, Bangalore, India; ${ }^{4}$ Junior Resident, Department of Obstetrics and Gynecology, Vydehi Institute of Medical Sciences and Research Centre, Whitefield, Bangalore, India.
} 